Published in final edited form as:

J Urol. 2018 October ; 200(4): 837-842. doi:10.1016/j.juro.2018.04.081.

\title{
Multi-Institutional Outcomes of Endoscopic Management of Stricture Recurrence after Bulbar Urethroplasty
}

\author{
Shyam Sukumar, \\ Department of Urology, University of Minnesota, Minneapolis, Minnesota \\ Sean P. Elliott, \\ Department of Urology, University of Minnesota, Minneapolis, Minnesota \\ Jeremy B. Myers, \\ Minneapolis, Minnesota, Department of Urology, University of Utah, Salt Lake City, Utah \\ Bryan B. Voelzke, \\ Department of Urology, University of Washington Medical Center, Seattle, Washington \\ Thomas G. Smith III, \\ Department of Urology, Baylor College of Medicine, Houston, Texas
}

\author{
Alexandra M. C. Carolan, \\ Department of Urology, University of Minnesota, Minneapolis, Minnesota \\ Michael Maidaa, \\ Department of Urology, University of lowa, lowa City, lowa
}

\author{
Alex J. Vanni, \\ Department of Urology, Lahey Hospital and Medical Center, Burlington, Massachusetts \\ Benjamin N. Breyer, and \\ Department of Urology, University of California-San Francisco, San Francisco, California \\ Bradley A. Erickson \\ Department of Urology, University of lowa, lowa City, lowa
}

\section{Abstract}

Purpose-Approximately $10 \%$ to $20 \%$ of patients will have a recurrence after urethroplasty. Initial management of these recurrences is often by urethral dilation or direct vision internal urethrotomy. In the current study we describe the outcomes of endoscopic management of stricture recurrence after bulbar urethroplasty.

Materials and Methods-We retrospectively reviewed bulbar urethroplasty data from 5 surgeons in the TURNS (Trauma and Urologic Reconstruction Network of Surgeons). Men who

\footnotetext{
"Correspondence: Department of Urology and Surgery, University of Iowa, 200 Hawkins Dr., Iowa City, Iowa 52242 (telephone: 319-356-7221; FAX: 319-356-3900; brad-erickson@uiowa.edu).

The corresponding author certifies that, when applicable, a statement(s) has been included in the manuscript documenting institutional review board, ethics committee or ethical review board study approval; principles of Helsinki Declaration were followed in lieu of formal ethics committee approval; institutional animal care and use committee approval; all human subjects provided written informed consent with guarantees of confidentiality; IRB approved protocol number; animal approved project number.
} 
underwent urethral dilation or direct vision internal urethrotomy for urethroplasty recurrence were identified. Recurrence was defined as the inability to pass a 17Fr cystoscope through the area of reconstruction. The primary outcome was the success rate of recurrence management. Comparisons were made between urethral dilation and direct vision internal urethrotomy, and then between endoscopic management of recurrences after excision and primary anastomosis urethroplasty vs substitutional repairs using time to event statistics.

Results-In 53 men recurrence was initially managed endoscopically. Median time to urethral stricture recurrence after urethroplasty was 5 months. At a median followup of 5 months the overall success rate was $42 \%$. Success after urethral dilation was significantly less than after direct vision internal urethrotomy ( 1 of 10 patients or $10 \%$ vs 21 of 43 or $49 \%, \mathrm{p}<0.001$ ) with a failure HR of 3.15 ( $\mathrm{p}=0.03$ ). Direct vision internal urethrotomy was more effective after substitutional failure than after excision and primary anastomosis urethroplasty ( $53 \%$ vs $13 \%, \mathrm{p}=0.005)$.

Conclusions-Direct vision internal urethrotomy was more successful than urethral dilation in the management of stricture recurrence after bulbar urethroplasty. Direct vision internal urethrotomy was more successful in patients with recurrence after substitution urethroplasty compared to after excision and primary anastomosis urethroplasty. Perhaps this indicates a different mechanism of recurrence for excision and primary anastomosis urethroplasty (ischemic) vs substitution urethroplasty (nonischemic).

\section{Keywords}

urethral stricture; ischemia; dilatation; anastomosis, surgical; recurrence

Male urethral stricture disease has an incidence rate of up to $0.6 \%$ with an estimated direct cost of care of more than $\$ 200$ million. ${ }^{1}$ While urethroplasty is widely regarded as the gold standard in the management of male urethral stricture disease, the overall recurrent stricture rate after urethroplasty is between $8.3 \%$ and $18.7 \%$ depending on repair type and location. ${ }^{2}$ While endoscopic options, including UD and/or DVIU, remain the most common initial management of these recurrences, the optimal treatment strategy in these patients remains unknown. Revision urethroplasty has a high success rate in this setting ${ }^{3-5}$ but it is significantly more invasive than endoscopic options.

In the current study we describe the outcomes of endoscopic management of recurrent stricture after bulbar urethroplasty. Our objective was to identify the best initial strategy of endoscopic treatment of patients with recurrence after urethroplasty.

\section{MATERIALS AND METHODS}

\section{Study Population}

Between 2007 and 2014 patients from 7 institutions affiliated with the TURNS were entered into an institutional review board approved database evaluating urethroplasty outcomes. The details of this prospective database were published previously. ${ }^{6}$ The current study includes cases of recurrent strictures after bulbar urethroplasty performed by 1 of 5 surgeons who contributed data to the combined web based database. Recurrent stricture management and outcomes data were available for all cases. The specific length of the recurrence was not 
recorded, although all substitution recurrences in this cohort developed at the distal and/or the proximal anastomosis and all recurrences after EPA were at the site of the original anastomosis. All EPAs were traditional transecting EPAs and nearly all substitution urethroplasties were performed with a buccal graft applied dorsally or ventrally.

\section{Urethroplasty Recurrence Definition}

We used the anatomical definition of stricture recurrence, which is that a standard $17 \mathrm{Fr}$ flexible cystoscope is unable to navigate past the area of reconstruction without force. ${ }^{7}$ Standard followup included 3 and 12-month cystoscopy, and cystoscopy yearly thereafter on an as needed basis. ${ }^{6}$

Men found to have anatomical stricture recurrence are generally offered endoscopic treatment prior to revision urethroplasty. However, men with asymptomatic recurrences will sometimes elect a watchful waiting strategy prior to any intervention, assuming that no active bladder and/or renal sequelae result. The percent of men who elected watchful waiting was determined in this study but specific followup details on these men were not analyzed.

The choice of urethral dilation or DVIU to manage recurrent stricture was left to surgeon discretion, and recurrent stricture length and caliber were not available in our database. All UDs were performed with a $24 \mathrm{Fr}$ balloon dilator (Cook Medical, Bloomington, Indiana or Boston Scientific, Marlborough, Massachusetts). These dilators are nonconforming and designed to handle pressures up to $20 \mathrm{~atm}$. All DVIUs were performed with a cold knife. Incisions were generally made in the spongy tissue at the 4 and 8 o'clock positions. However, because recurrence characteristics are heterogeneous, other incisions were also made to accommodate stricture specific characteristics at surgeon discretion. All endoscopic procedures were followed by a short catheterization period of 3 to 7 days.

Routine followup after endoscopic repair included cystoscopy at 3 months and then every 6 months until stricture stabilization or a need for repeat urethroplasty was determined.

Notably this differed from our cystoscopic followup after primary urethroplasty, given the higher suspected chance of recurrence.

\section{Statistical Analysis}

Our primary outcome was success after endoscopic treatment of stricture recurrence, defined as the ability to traverse the endoscopically managed recurrent stricture with a cystoscope without force. Subanalyses included 1) a comparison of outcomes between DVIU and UD, and 2) a comparison of DVIU outcomes for EPA and substitution recurrences. Time to event analysis was performed with Kaplan-Meier curves ${ }^{8}$ and Cox regression models. ${ }^{9}$ Statistical significance was considered at $\mathrm{p}<0.05$. All statistical analyses were performed with IBM ${ }^{\circledR}$ SPSS ${ }^{2} 20$.

\section{RESULTS}

\section{Demographics}

A total of 130 men experienced recurrence after urethroplasty, of whom $53(41 \%)$ elected initial endoscopic management of the recurrence. Those men were the focus of this study. Of 
these cases 23\% developed after EPA and 77\% developed after substitution urethroplasty. Notably only 4 patients $(7.5 \%)$ who elected endoscopic management of recurrence were completely asymptomatic at the time of routine cystoscopy. Median time to urethral stricture recurrence after urethroplasty was 5 months (IQR 4-12).

Initial endoscopic treatment was DVIU in 43 men (81\%) and urethral dilation in 10 (19\%). All endoscopic procedures were performed with curative intent and none was augmented with an injection of steroid or another injectable meant to modify recurrence.

\section{Direct Visual Internal Urethrotomy versus Urethral Dilation}

At a median followup of 5 months (IQR 2.1-23) $41.5 \%$ of patients in the overall cohort were free of recurrence after endoscopic treatment. The DVIU success rate was statistically higher than that of UD $(48.8 \%$ vs $10 \%, \mathrm{p}<0.0001)$.

Time to event analysis revealed significant differences in the recurrence rate between UD and DVIU (log rank p <0.001, fig. 1). Cox modeling revealed a threefold higher risk of failure for UD than for DVIU (HR 3.15, 95\% CI 1.07-9.29, $\mathrm{p}=0.038$ ) and a twofold higher but nonsignificant risk in patients who underwent EPA vs substitution urethroplasty (HR $2.41,95 \%$ CI $0.99-5.85, \mathrm{p}=0.053)$.

\section{Direct Visual Internal Urethrotomy Success for Excision and Primary Anastomosis Urethroplasty versus Substitution Failures}

The success rate of DVIU for substitution repairs was significantly higher than that for EPA failures (53\% vs 13\%). On time to event analysis DVIU was more successful after substitution urethroplasty than after EPA (log rank $\mathrm{p}=0.005$, fig. 2).

\section{DISCUSSION}

Primary endoscopic treatment and urethroplasty are the major treatment options in patients with anterior urethral stricture disease with the latter considered the current gold standard in men with bulbar urethral strictures. ${ }^{10-12}$ There is a high level of evidence that the 2 modalities of primary endoscopic management (UD and DVIU) have equal efficacy. ${ }^{13,14}$ This equivalency was reiterated in the AUA (American Urological Association) guideline on the management of male urethral strictures. ${ }^{15}$ However, recurrent stricture disease after primary urethroplasty is considered mechanistically different in origin and it is unclear whether the mentioned equivalency extends to such patients. Specifically recurrent urethral strictures after urethroplasty tend to be more focal (at the site of previous anastomoses) and have less associated spongiofibrosis and better overall vascularity than primary urethral strictures. ${ }^{16}$

In the current study we evaluated the outcomes of endoscopic treatment in patients with recurrence after initial bulbar urethroplasty. We found that at a mean followup of 16 months patients treated with DVIU for recurrent stricture disease after urethroplasty had a statistically significantly higher success rate than patients who underwent urethral dilation (48.8\% vs 10\%). In a subset of patients treated with DVIU after urethroplasty failure DVIU after substitution urethroplasty was more successful than after EPA. 
While this study was not specifically designed to elucidate the reasons for differences in the success rate of endoscopic failure management, we believe that a plausible explanation may exist which could affect clinical practice. EPA involves excision of the diseased segment of the urethra and reanastomosis of healthy proximal and distal segments. This anastomosis should be under no tension and in a spatulated manner, allowing for moderate postprocedural lumen contraction without affecting the final lumen size. Therefore, assuming that the procedure was technically appropriate and all diseased urethral tissue was appropriately excised, failed repair is more likely to be related to postoperative local ischemia and, thus, it will not respond well to endoscopic repair.

On the other hand, substitution repair is quite different in that urethral transection typically does not occur. This theoretically would better preserve the local blood supply and make local ischemia a less likely cause of failure. Rather, technical concerns at the anastomotic sites and/ or under recognition of the stricture extent may be more plausible causes. Thus, an intact spongiosum at the site of failed substitution urethroplasty may portend better outcomes of endoscopic management than the failures after EPA.

Of note our $42 \%$ success rate of endoscopic management after urethroplasty failure was lower than the $50 \%$ to $70 \%$ in earlier reports. ${ }^{17-19}$ However, these reports were single institution series, they had a small sample size of recurrence or there was no standardized definition of treatment failure. To our knowledge the current report is the largest multiinstitutional analysis of the outcomes of initial endoscopic treatment in patients with recurrence after initial urethroplasty.

This study has limitations that deserve special mention. 1) This is a retrospective study of prospectively entered data and so was subject to the biases pertinent to such studies. ${ }^{20}$

2) While the overall number of patients was small at 53 and median followup was only 5 months, this is to our knowledge the largest multi-institutional study of endoscopic management of urethroplasty recurrence.

3) The choice of whether to perform DVIU vs UD was at surgeon discretion. As described these procedures were previously found to have similar efficacy. However, given the findings in the current study, future studies of endoscopic management after urethroplasty should account for different success rates of DVIU vs dilation.

4) Not all patients with anatomical recurrence underwent treatment since many were asymptomatic (ie there was no functional recurrence). Thus, there was some selection bias and it is unclear how this affected outcomes.

5) We did not have information on recurrent stricture characteristics, including length or caliber, which may have offered even more insight into which types of recurrence were likely to be amenable to endoscopic repair.

6) While we postulated a different mechanism of failure after DVIU vs dilation, we did not have histological data such as a tissue diagnosis that might have helped elucidate tissue level metrics. 


\section{CONCLUSIONS}

DVIU is superior to urethral dilation for the management of stricture recurrence after bulbar urethroplasty. DVIU is more successful in patients with recurrence after substitution urethroplasty than after EPA, perhaps indicating a different mechanism of recurrence for EPA (ischemic) vs substitution urethroplasty (nonischemic).

\section{Abbreviations and Acronyms \\ DVIU direct vision internal urethrotomy \\ EPA excision and primary anastomosis urethroplasty \\ UD urethral dilation}

\section{REFERENCES}

1. Santucci RA, Joyce GF and Wise M: Male urethral stricture disease. J Urol 2007; 177: 1667. [PubMed: 17437780]

2. Meeks JJ, Erickson BA, Granieri MA et al.: Stricture recurrence after urethroplasty: a systematic review. J Urol 2009; 182: 1266. [PubMed: 19683309]

3. Javali TD, Katti A and Nagaraj HK: Management of recurrent anterior urethral strictures following buccal mucosal graft-urethroplasty: a single center experience. Urol Ann 2016; 8: 31. [PubMed: 26834398]

4. Levine MA, Kinnaird AS and Rourke KF: Revision urethroplasty success is comparable to primary urethroplasty: a comparative analysis. Urology 2014; 84: 928. [PubMed: 25129537]

5. Blaschko SD, McAninch JW, Myers JB et al.: Repeat urethroplasty after failed urethral reconstruction: outcome analysis of 130 patients. J Urol 2012; 188: 2260. [PubMed: 23083654]

6. Erickson BA, Elliott SP, Voelzke BB et al.: Multi-institutional 1-year bulbar urethroplasty outcomes using a standardized prospective cystoscopic follow-up protocol. Urology 2014; 84: 213. [PubMed: 24837453]

7. Erickson BA and Ghareeb GM: Definition of successful treatment and optimal follow-up after urethral reconstruction for urethral stricture disease. Urol Clin North Am 2017; 44: 1. [PubMed: 27908363]

8. Kaplan EL and Meier P: Nonparametric estimation from incomplete observations. J Am Statist Ass 1958; 53: 457.

9. Cox DR: Regression models and life-tables. J Roy Statist Soc Series B 1972; 34: 187.

10. Buckley JC, Heyns C, Gilling P et al.: SIU/ICUD Consultation on Urethral Strictures: dilation, internal urethrotomy, and stenting of male anterior urethral strictures. Urology, suppl., 2014; 83: S18. [PubMed: 24286602]

11. Morey AF, Watkin N, Shenfeld O et al.: SIU/ICUD Consultation on Urethral Strictures: anterior urethra-primary anastomosis. Urology, suppl., 2014; 83: S23. [PubMed: 24373726]

12. Chapple C, Andrich D, Atala A et al.: SIU/ICUD Consultation on Urethral Strictures: the management of anterior urethral stricture disease using substitution urethroplasty. Urology, suppl., 2014; 83: S31. [PubMed: 24411214]

13. Steenkamp JW, Heyns CF and de Kock ML: Internal urethrotomy versus dilation as treatment for male urethral strictures: a prospective, randomized comparison. J Urol 1997; 157: 98. [PubMed: 8976225]

14. Wong SS, Aboumarzouk OM, Narahari R et al.: Simple urethral dilatation, endoscopic urethrotomy, and urethroplasty for urethral stricture disease in adult men. Cochrane Database Syst Rev 2012; 12: CD006934. [PubMed: 23235635]

15. Wessells H, Angermeier KW, Elliott S et al.: Male urethral stricture: American Urological Association guideline. J Urol 2017; 197: 182. [PubMed: 27497791] 
16. Voelzke BB and Wessells H: Role of direct vision internal urethrotomy in the management of urethral stricture. AUA Update Series 2010; vol 29, lesson 20.

17. Elliott SP, Metro MJ and McAninch JW: Long-term followup of the ventrally placed buccal mucosa onlay graft in bulbar urethral reconstruction. J Urol 2003; 169: 1754. [PubMed: 12686826]

18. Brown ET, Mock S, Dmochowski R et al.: Direct visual internal urethrotomy for isolated, posturethroplasty strictures: a retrospective analysis. Ther Adv Urol 2017; 9: 39. [PubMed: 28203286]

19. Rosenbaum CM, Schmid M, Ludwig TA et al.: Internal urethrotomy in patients with recurrent urethral stricture after buccal mucosa graft urethroplasty. World J Urol 2015; 33: 1337. [PubMed: 25428791]

20. Ward RA and Brier ME: Retrospective analyses of large medical databases: what do they tell us? J Am Soc Nephrol 1999; 10: 429. [PubMed: 10215345] 


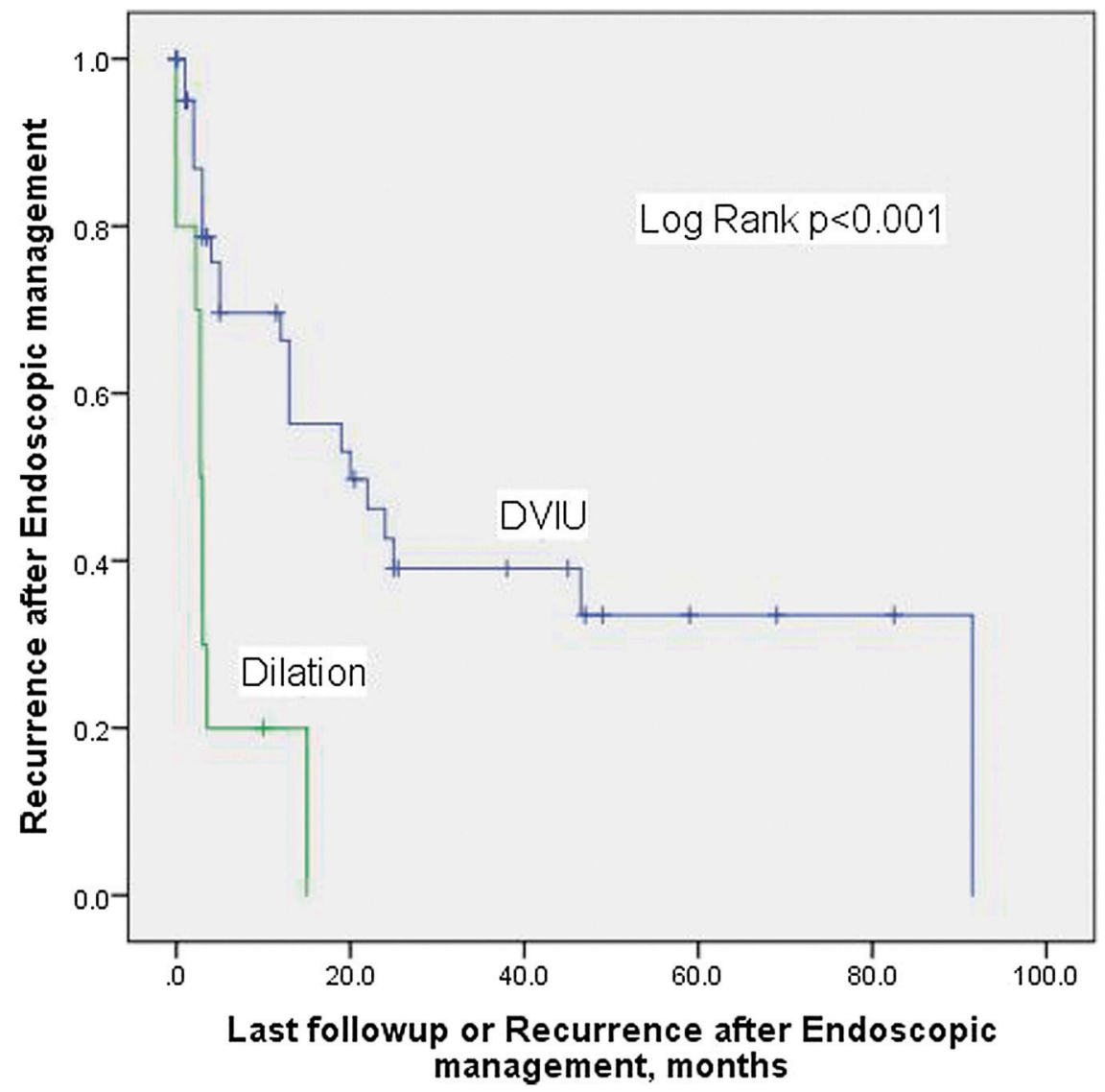

Figure 1.

Kaplan-Meier analysis of failures after endoscopic management of urethroplasty recurrences stratified by endoscopic management type. 


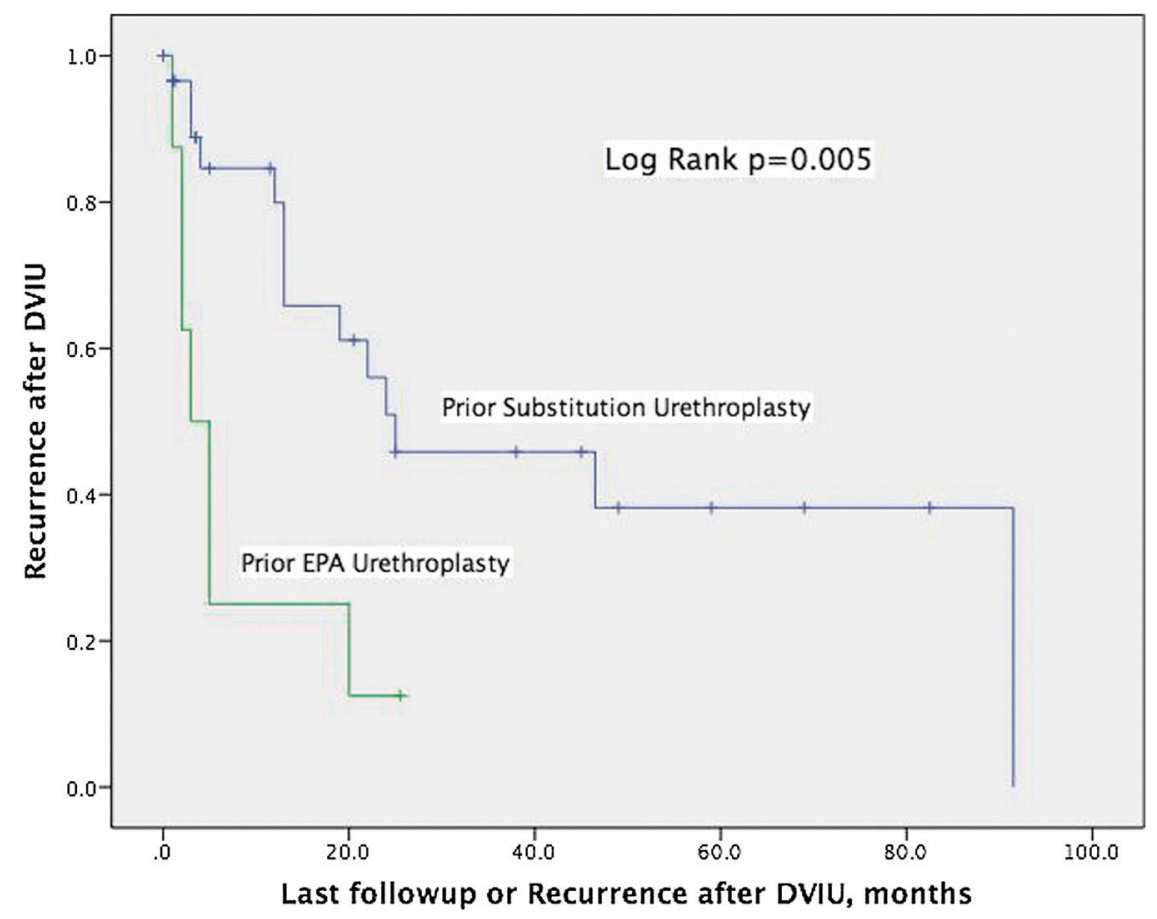

Figure 2.

Kaplan-Meier analysis of failures after direct vision internal urethrotomy performed as initial management of urethroplasty recurrences stratified by primary urethroplasty type. 